Article

\title{
Bmserpin2 Is Involved in BmNPV Infection by Suppressing Melanization in Bombyx mori
}

\author{
Shahzad Toufeeq ${ }^{1,2,+}$, Jie Wang ${ }^{1,2,+}$, Shang-Zhi Zhang ${ }^{1,2}$, Bing $\mathrm{Li}^{1,2}$, Pei Hu ${ }^{1,2}$, Lin-Bao Zhu ${ }^{1,2}$, \\ Ling-Ling You ${ }^{1,2}$ and Jia-Ping $X u^{1,2, *}$ \\ 1 School of Life Sciences, Anhui Agricultural University, Hefei 230036, China; toufeeqphd@ahau.edu.cn (S.T.); \\ wangjie_3001@163.com (J.W.); 18755148780@163.com (S.-Z.Z.); libing2504@sina.com (B.L.); \\ m15755074091@163.com (P.H.); zhulinbao@163.com (L.-B.Z.); 13063306714@163.com (L.-L.Y.) \\ 2 Anhui International Joint Research and Developmental Center of Sericulture Resources Utilization, \\ Hefei 230036, China \\ * Correspondence: jiapingxu@163.com \\ + These authors contributed equally to this work.
}

Received: 23 September 2019; Accepted: 6 November 2019; Published: 11 November 2019

check for updates

\begin{abstract}
Melanization, an important defense response, plays a vital role in arthropod immunity. It is mediated by serine proteases (SPs) that convert the inactive prophenoloxidase (PPO) to active phenoloxidase (PO) and is tightly regulated by serine protease inhibitors (serpins) which belong to a well distributed superfamily in invertebrates, participating in immune mechanisms and other important physiological processes. Here, we investigated the Bmserpin2 gene which was identified from a transcriptome database in response to Bombyx mori nucleopolyhedrovirus (BmNPV) infection. Quantitative real-time polymerase chain reaction (qRT-PCR) results showed that Bmserpin2 was expressed in all tissues, with maximum expression in fat body. Upon BmNPV infection, the expression of Bmserpin2 was up-regulated in P50 (susceptible strain) and BC9 (resistant strain) in haemocytes, fat body and the midgut. However, up-regulation was delayed in BC9 (48 or $72 \mathrm{~h}$ ), in contrast to P50 (24 h), after BmNPV infection. Meanwhile, Bmserpin2 could delay or inhibit melanization in silkworm haemolymph. Significant increased PO activity can be observed in Bmserpin2-depleted haemolymph under NPV infection. Furthermore, the viral genomic DNA copy number was decreased in Bmserpin2-depleted haemolymph. We conclude that Bmserpin2 is an inducible gene which might be involved in the regulation of PPO activation and suppressed melanization, and have a potential role in the innate immune system of $B$. mori.
\end{abstract}

Keywords: Bombyx mori; NPV; Serpin2; melanization

\section{Introduction}

Bombyx mori is a well-known lepidopteran insect with a great economic value as a producer of silk. B. mori is also used in many studies as model insect in genetics and applied biotechnology [1]. Bombyx mori nucleopolyhedrovirus (BmNPV) is a major burden for silkworms that causes serious loss to the sericulture industry. BmNPV contains two types of virion phenotypes, budded virus (BV) and occlusion-derived virus (ODV). BV transfects cell-to-cell, while ODV spreads from one host to another host [2]. Most B. mori strains are highly susceptible to BmNPV, while only a few are highly resistant [3]. Studies on susceptible and resistant strains have increased the understanding of the mechanisms triggered by virus infection, however, a detailed understanding of $B$. mori resistance to BmNPV infection is yet elusive [4].

Insects solely depend on their innate immunity which comprises of humoral and cellular responses to combat pathogens [5,6]. Besides humoral and cellular responses, intracellular responses, such as 
apoptosis, RNAi and melanization also contribute to insect defenses [7]. Melanization is an important immune component in the insect defence system and is stimulated by the serine proteases (SPs) cascade that converts inactive prophenoloxidase (PPO) to active phenoloxidase (PO). This leads to the synthesis of quinones and melanin which encapsulates the invading pathogens [8-10]. For successful elimination of pathogens, expression of serine proteases (SPs), and their activation is tightly regulated by serine protease inhibitors (serpins), which are the largest known superfamily of protease inhibitors in vertebrates and invertebrates. A typical serpin structure contains a serpin domain and a 20-amino-acid reactive centre loop (RCL) at the C-terminus, acting as "bait" for target proteases [11]. During the inhibition process, serpin interacts with its target protease, and uses its RCL to bait the protease and go through dramatic conformational change which eventually inhibit the protease [12,13].

Insect serpins are key players in the defense mechanism of insects, especially the Toll pathway and PPO cascade [14]. Due to their crucial role in insect immunity, serpins have been widely investigated in several insects that are model organisms and/or agricultural pests, including Drosophila melanogaster [15], Bombyx mori [16], Manduca sexta [17], Helicoverpa armigera [18], Tenebrio molitor [19], and Tribolium castaneum [20]. Biochemical studies revealed that serpins are negative regulators of PPO. For example, in M. sexta serpin-1J, serpin-3, serpin-6, serpin-7 and serpin-12 negatively regulate PPO cascade via inhibiting proteases [8,21-23]. Several serpins including serpin-5, serpin-6, serpin-15, serpin-18, and serpin-32 from Bombyx mori have proved to negatively affect the PPO pathway [24-28].

Recent studies have suggested that melanization is involved in combating virus infection in larval insects. For instance, in tobacco budworm, haemolymph acts as a viricide [29]. Moreover, 5,6-dihydroxyindole (DHI), a melanin precursor, has broad-spectrum antiviral activity [30]. Also, the PO cascade in Aedes aegypti blocked Semliki Forest virus (SFV) infection [31]. Yuan et al. revealed that serpin-5 and serpin-9 regulate melanization and promote baculovirus infection [32]. However, there have been very few studies on silkworm serpins in response to BmNPV infection.

To better understand the silkworm serpins and melanization mechanism, we studied B. mori serpin-2 (Bmserpin2) under BmNPV infection. We found that Bmserpin2 can be induced by BmNPV infection, and knockdown of serpin-2 increases $\mathrm{PO}$ activity. This study should support further study on the serpins in response to BmNPV infection.

\section{Materials and Methods}

\subsection{Rearing of Silkworm and B. mori Nucleopolyhedrovirus (BmNPV) Preparation}

The B. mori susceptible strain (P50) and resistant strain (BC9) were preserved in the Anhui International Joint Research and Development Centre of Sericulture Resources Utilization, Anhui Agricultural University, Hefei, China. BC9 is a near-isogenic strain which was obtained when P50 and A35 (a highly resistant strain to BmNPV) were crossed, and offspring were repeatedly backcrossed with P50 for nine generations to construct BC9, and each offspring was screened for BmNPV [33]. Larvae were reared using fresh mulberry leaves at $26 \pm 1{ }^{\circ} \mathrm{C}, 75 \pm 5 \%$ relative humidity with $12 \mathrm{~h}$ day/night cycles. The BmNPV T3 strain was maintained and purified as described previously [34].

\subsection{BmNPV Infection to B. mori}

Virus infection was carried out according to previous published study [35]. Briefly, P50 and BC9 (3rd day fifth instar) larvae were fed orally with $5 \mu \mathrm{L}$ OBs $\left(1 \times 10^{6} \mathrm{OBs} / \mathrm{mL}\right.$ in water), while each larva in the control group was administrated with $5 \mu \mathrm{L}$ of water. Normal feeding continued after infection. The insects were sacrificed, and different tissues (haemocytes, fat body and the midgut) were collected at different time points after infection. The samples were frozen in liquid $\mathrm{N}_{2}$ and stored at $-80{ }^{\circ} \mathrm{C}$ for later use. 


\subsection{Total RNA Isolation and Amplification of BmSerpin2}

Total RNA from silkworm tissues was extracted by Trizol reagent (Takara, Dalian, China), and first-strand cDNA was synthesised using the PrimeScript RT kit (TaKaRa, Dalian, China) according to the manufacturer's instructions. Bmserpin2 was identified from a previous study [33], and gene specific primers (Table 1) were designed with the Primer premier 5.0 software and used to amplify the Bmserpin2 open reading frame (ORF). A polymerase chain reaction (PCR) was carried out as follows: pre-denaturation at $94^{\circ} \mathrm{C}$ for $5 \mathrm{~min}$; 35 cycles at $94{ }^{\circ} \mathrm{C}$ for $30 \mathrm{~s}, 55^{\circ} \mathrm{C}$ for $30 \mathrm{~s}$, and $72{ }^{\circ} \mathrm{C}$ for $1 \mathrm{~min} 20 \mathrm{~s}$ and $72{ }^{\circ} \mathrm{C}$ for $10 \mathrm{~min}$ for extension. The PCR products were resolved on $1 \%$ agarose gel and purified using a DNA Purification Kit (Tiangen Biotech, Beijing, China). Purified PCR products were cloned into the pMD19-T vector and sequenced to verify the cloned fragments.

Table 1. The primers used in this study.

\begin{tabular}{ccc}
\hline Primers & Sequences of Primers $\left(\mathbf{5}^{\prime}\right.$ to $\mathbf{3}^{\prime} \mathbf{)}$ & Purpose \\
\hline Bmserpin2-F & CGCGGATCCATGATATTTGCCAGAGTTTTGTT & Protein expression \\
Bmserpin2- $R$ & CCGCTCGAGTCAATTTCGTCCACGGTATT & Protein expression \\
Bmserpin2-F & CAGAATACTTGCTGGCGTTGATA & qRT-PCR \\
$B m s e r p i n 2-R$ & AGCCGAGAATGATGAGCGAAT & qRT-PCR \\
$B m$ GAPDH-F & CATTCCGCGTCCCTGTTGCTAAT & qRT-PCR \\
$B m G A P D H-R$ & GCTGCCTCCTTGACCTTTTGC & qRT-PCR \\
GP64- $F$ & GAAGTAGAAACGCCGCATCG & qRT-PCR \\
GP64- $R$ & GTGGGGTATTCAGGCAGCAGT & qRT-PCR \\
\hline
\end{tabular}

The restriction enzyme sites are marked in underline.

\subsection{Recombinant Bmserpin2 and Antibody Preparation}

The cloned cDNA fragment that contained mature Bmserpin2 ligated into the expression vector pET-28a (Novagen) with restriction enzyme sites BamH1 and Xho1, and the insertion was confirmed by DNA sequencing in the recombinant BmSerpin2-pET-28a plasmid and then the recombinant plasmid was transformed into Escherichia coli BL21 (Novagen) competent cells which were grown in fresh Luria-Bertani (LB) medium. The positive plasmid was induced by isopropyl $\beta$-D-thiogalactoside (IPTG) $(1 \mathrm{mM})$ to induce recombinant Bmserpin2 expression for $6 \mathrm{~h}$ at $37^{\circ} \mathrm{C}$. After centrifugation at $7500 \times \mathrm{g}$ for $10 \mathrm{~min}$ at $4{ }^{\circ} \mathrm{C}$, bacteria were collected and sonicated in phosphate-buffered saline (PBS). The insoluble pellet was dissolved in buffer A (100 mM NaH${ }_{2} \mathrm{PO}_{4}, 10 \mathrm{mM}$ Tris- $\mathrm{HCl}, 8 \mathrm{M}$ urea, pH 8.0). Bmserpin2 protein was purified using a Ni-NTA Fast Start Kit (Qiagen, Inc., Valencia, CA, USA) according to the manufacturer's protocol. After purification, the protein was analysed by $12 \%$ sodium dodecyl sulfate polyacrylamide gel electrophoresis (SDS-PAGE) and western blotting using anti-His-antibody. The concentration of Bmserpin2 protein was determined by using the BCA protein Assay Kit (Novagen, Hilden, Germany). The polyclonal antibody of Bmserpin2 was prepared by HuaAn Biotechnology Co., Ltd. (Hangzhou, China).

\subsection{Preparation of Soluble Bmserpin2}

To perform the biochemical analysis of Bmserpin2, the purified recombinant Bmserpin2 was renatured through a dialysis method. $3 \mathrm{~mL}$ of Bmserpin $2(0.2 \mathrm{mg} / \mathrm{mL})$ was loaded into the dialysis bag (Sangon Biotech, Shanghai, China). Bmserpin2 was dialysed in the refolding buffer (5\% glycerol, $1 \%$ arginine, $2 \%$ glycine and the following urea gradient: $6 \mathrm{M}, 4 \mathrm{M}, 3 \mathrm{M}, 2 \mathrm{M}, 1 \mathrm{M}, 0 \mathrm{M}$ ) and rotated on ice from high to low urea gradients for $2 \mathrm{~h}$ per gradient. After dialysis, the protein was centrifuged at $12,000 \times \mathrm{g}$ for $10 \mathrm{~min}$ at $4{ }^{\circ} \mathrm{C}$. The supernatant was collected, and ultrafiltration was carried out using centrifugal filter devices (Millipore, Bedford, MA, USA). The protein was saved and used for later experiments. 


\subsection{Expression Analysis of Bmserpin2 in Different Tissues and Developmental Stages}

Total RNA was extracted from different tissues (midgut, fat body, haemocytes, integument, Malpighian tubules, head and silk gland) and developmental stages (egg to adult) using Trizol reagent (Invitrogen, Grand Island, NY, USA) according to the manufacturer's instructions. The concentration and integrity of RNA were observed at an absorbance ratio of $\mathrm{A}_{260 / 280}$ and $\mathrm{A}_{260 / 230}$ using a NanoDrop 2000 spectrophotometer (Thermo Scientific ${ }^{\mathrm{TM}}$, Waltham, MA, USA), and by 1.0\% agarose gel electrophoresis, respectively. Total RNA samples were used to prepare first strand cDNA using PrimeScript $^{\text {TM }}$ RT Master Mix (Takara, Dalian, China) following the manufacturer's instructions. The qRT-PCR was performed with a TB Green kit (Takara) and analyzed with the Multicolour Real-time PCR Detection System (Bio-Rad, Hercules, CA, USA), as previously reported [36]. The thermal cycling profile was set as follows: initial denaturation at $95^{\circ} \mathrm{C}$ for $30 \mathrm{~s}$ and 40 cycles of $95{ }^{\circ} \mathrm{C}$ for $5 \mathrm{~s}, 60^{\circ} \mathrm{C}$ for $30 \mathrm{~s}$, and $72{ }^{\circ} \mathrm{C}$ for $20 \mathrm{~s}$. The transcriptional level of Bmserpin 2 was normalized to B. mori GAPDH. The relative expression levels were calculated using the $2^{-\Delta \Delta \mathrm{Ct}}$ method according to a previous protocol [37]. The experiment was conducted in triplicate.

\subsection{Expression Analysis of Bmserpin2 after BmNPV Infection}

To determine the expression of Bmserpin2 after BmNPV infection, different tissues including haemocytes, fat body and the midgut were collected from P50 and BC9 strains at 6, 12, 24, 48 and $72 \mathrm{~h}$ after infection. Total RNA was extracted and cDNA was prepared as described above. The mRNA level of Bmserpin2 was analysed by qRT-PCR. The transcriptional level of Bmserpin2 was normalized to B. mori GAPDH. The experiment was conducted in triplicate.

\subsection{Western Blotting}

Proteins were extracted from the midgut at $24 \mathrm{~h}$ post infection (hpi) and haemolymph (24, 48 and $72 \mathrm{hpi}$ ) of P50 and BC9 strains. $1 \mathrm{~mL}$ of protein lysis buffer (4\% CHAPS, $2 \mathrm{M}$ thiourea, $7 \mathrm{M}$ urea), $0.12 \mathrm{~g}$ samples of midgut or $500 \mu \mathrm{L}$ samples of haemolymph, $1 \mathrm{mM}$ phenyl methane sulfonyl fluoride (PMSF) and $10 \mathrm{mg}$ of dithiothreitol (DTT) were added into glass homogenisers for homogenising. After centrifugation at $12,000 \times g$ at $4{ }^{\circ} \mathrm{C}$ for $40 \mathrm{~min}$, protein concentrations were determined by BCA. The extracted protein $(25 \mu \mathrm{g})$ were loaded on SDS-PAGE and transferred to polyvinylidene fluoride (PVDF) membrane, and incubated with the anti-Bmserpin2 (1:500) primary antibody. GAPDH antibody (1:1000) was used as a reference. Horseradish peroxidase (HRP)-conjugated goat anti-rabbit and goat anti-mouse (Transgene Biotech, Beijing, China) (1:5000 dilution) were used as secondary antibodies.

\subsection{Effect of Bmserpin2 on the Melanization of Haemolymph}

To observe the effect of Bmserpin2 on the melanization of the haemolymph, a previous protocol was followed with a few modifications [28]. Briefly, the cell-free haemolymph from the 3rd day fifth instar larvae was collected. The sample $(5 \mu \mathrm{L})$ was incubated with $0,1,2,4$ and $6 \mu \mathrm{g}$ of soluble prepared Bmserpin2 diluted in PBS. The reaction was performed at room temperature and captured by camera to observe colour difference in samples at $0,5,10$ and $20 \mathrm{~min}$.

\subsection{Synthesis and Micro-Injection of Small Interfering RNA (siRNA)}

RNAi experiment was performed by following previous protocol [38]. In short, siRNA sequences were obtained from two different regions of the Bmserpin2 gene using an online tool (http://bioinfo.clontech.com/rnaidesigner/sirnaSequenceDesignInit.do). The siRNAs were synthesised by GenePharma Technology Co. Ltd. (Shanghai, China). Table 2 shows the siRNA sequences. The siRNAs were injected to 3rd day of 5th instar larvae. Each larva of the siRNA group was injected with $2 \mu \mathrm{L}(2 \mu \mathrm{g} / \mu \mathrm{L})$ of siRNA solution. Larvae in the control group were injected with same dose of si green fluorescent protein (siGFP) as control. After $24 \mathrm{~h}$ of the injection of siRNAs, larvae were infected with BmNPV for downstream experiments. The experiment was conducted in triplicate. 
Table 2. Sequences of siRNA against Bmserpin2.

\begin{tabular}{ccc}
\hline Name & \multicolumn{1}{c}{ Sense Strand } & Antisense Strand \\
\hline siRNA-1 & GGAAAUAAAGAACCCUGUUTT & AACAGGGUUCUUUAUUUCCTT \\
siRNA-2 & GCUGCCGAACGAAAUUAAUTT & AUUAAUUUCGUUCGGCAGCTT \\
siGFP & UUCUCCGAACGUGUCACGUTT & ACGUGACACGUUCGGAGAATT \\
\hline
\end{tabular}

\subsection{Phenoloxidase (PO) Activity Assay}

The PO activity assay was conducted using a previously described protocol [32]. Briefly, cell-free haemolymph samples were collected from P50 at $48 \mathrm{~h}$ after BmNPV infection without adding phenylthiourea (PTU). Samples were incubated at $27^{\circ} \mathrm{C}$ for $30 \mathrm{~min}$ and the PO activity assay was conducted in 96-well plates containing $1 \mu \mathrm{L}$ haemolymph followed by the addition of $200 \mu \mathrm{L}$ substrate solution ( $2 \mathrm{mM}$ dopamine in $50 \mathrm{mM}$ sodium phosphate buffer ( $\mathrm{pH}$ 6.5). PO activity was measured at $490 \mathrm{~nm}$ in a microtiter plate reader. One unit of PO activity was defined as $\Delta 490$ of 0.001 in one minute. The PO activity of siRNA treated cell-free haemolymph of Bmserpin 2 were performed in the same way as described above.

\subsection{Examination of Viral DNA in Haemolymph}

The virus genomic DNA from Bmserpin2 depleted haemolymph was extracted by following previous protocols [39]. Briefly, haemolymph $(100 \mu \mathrm{L})$ was mixed with $100 \mu \mathrm{L}$ of $1 \mathrm{M} \mathrm{NaOH}$, and incubated at room temperature for $5 \mathrm{~min}$, then $20 \mu \mathrm{L}$ of ammonium acetate was added and samples were incubated again for $5 \mathrm{~min}$. An equal volume of phenol/chloroform (1:1) was used for extraction and samples were centrifuged at $12,000 \times g$ for $10 \mathrm{~min}$. The supernatant was collected and precipitated in absolute ethanol. The final pellet was resuspended in $20 \mu \mathrm{L}$ TE buffer. qRT-PCR was carried out to measure the viral genomic DNA copies using BmNPV GP64. B. mori GAPDH was used to normalize the expression of target gene. The assay was performed in triplicate.

\section{Results}

\subsection{Tissue Distribution and Expression of BmSerpin2 Gene at Different Developmental Stages}

The tissue distribution and developmental stages of Bmserpin2 expression levels were analysed by qRT-PCR. The results indicated that Bmserpin 2 was transcribed in all tissues including the midgut, fat body, haemocytes, integument, Malpighian tubules and head and silk gland. However, the transcriptional level of Bmserpin 2 was markedly higher in the fat body, and the lowest expression was observed in the silk gland (Figure 1). Furthermore, the developmental stages result revealed that the expression level of Bmserpin 2 was the highest in fifth instar larvae, and the expression in larvae was higher than in pupae and adults (Figure 2). 


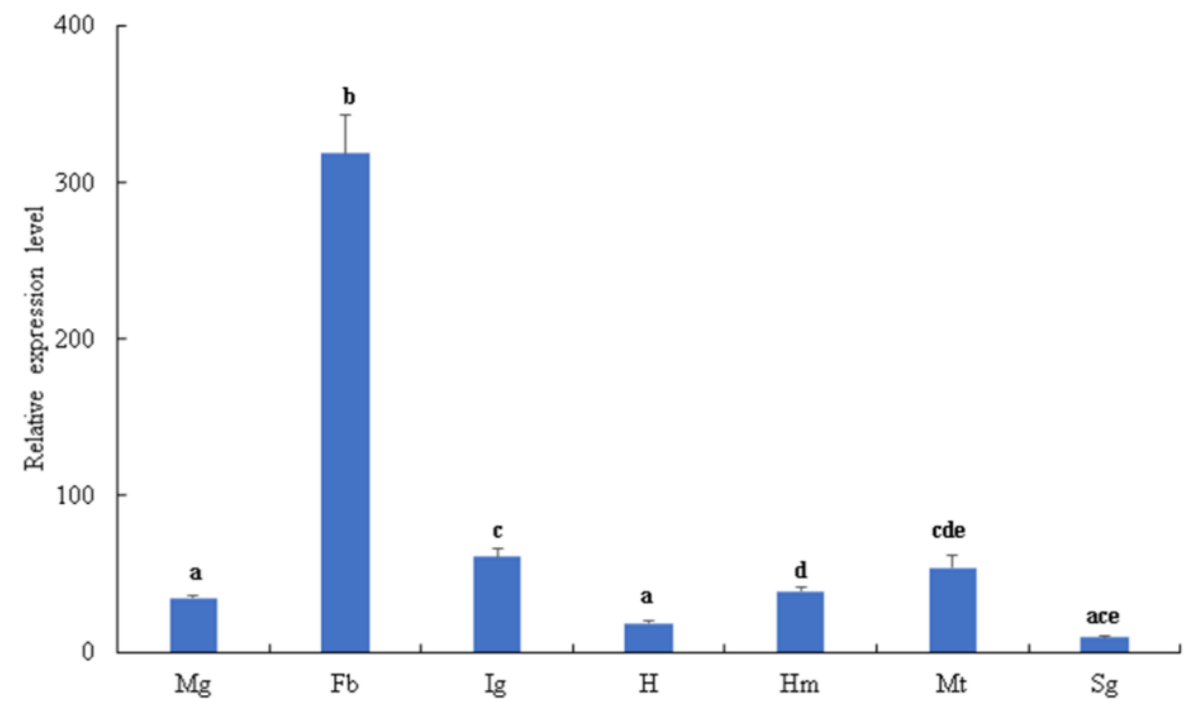

Figure 1. Tissue distribution of Bmserpin2 in the 5th instar B. mori larvae. The mRNA levels of Bmserpin2 were analysed by qRT-PCR. The Bmserpin 2 transcript level in the midgut was used as the calibrator. Data were normalised with Bombyx mori glyceraldehyde 3-phosphate dehydrogenase (GAPDH). Error bars show mean \pm standard deviation (SD, $n=3$ ). Significant difference is indicated with different letters (one-way ANOV followed by Tukey's test, $p<0.05$ ). Mg: Midgut, Fb: Fat body, Ig: Integument, H: Head, Hm: Haemolymph, Mt: Malpighian tubule, Sg: Silk gland.

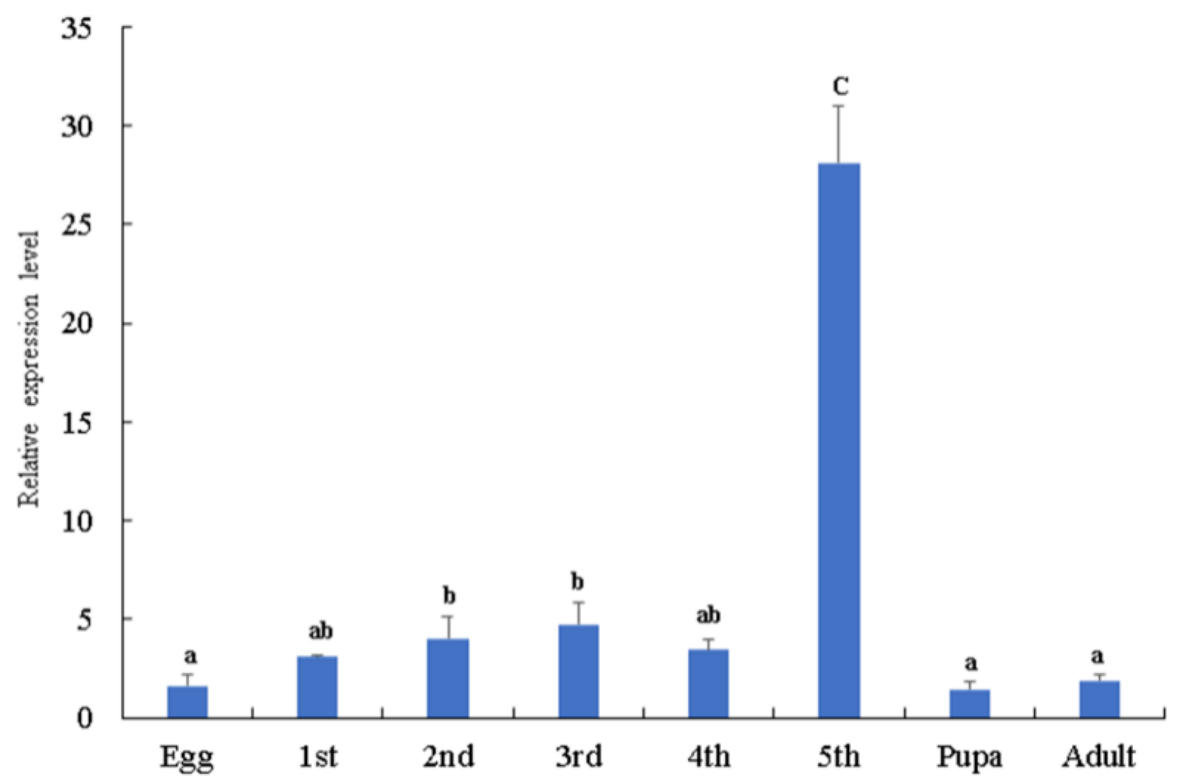

Figure 2. The expression pattern of Bmserpin 2 in different developmental stages using whole insect body. Analysis of mRNA levels of Bmserpin2 in different developmental stages using qRT-PCR. The Bmserpin2 transcript level in the egg was used as the calibrator. Data were normalised with Bombyx mori GAPDH. Error bars show mean $\pm \mathrm{SD}(n=3)$. Significant difference is indicated with different letters (one-way ANOVA followed by Tukey's test, $p<0.05)$.

\subsection{Expression Analysis of Bmserpin2 in Response to BmNPV Infection}

To investigate the mRNA expression level of Bmserpin2 in response to BmNPV infection, silkworm P50 and BC9 strains were used. Temporal expression was analysed by qRT-PCR in the haemocytes, fat body and midgut. Our results suggested that compared with P50- (control), Bmserpin2 mRNA expression in P50+ (infected) was significantly increased after 24, 48 and 72 hpi in the haemocytes (Figure 3A). In the fat body, Bmserpin2 expression varied after post-infection, however, its expression 
was highest at $24 \mathrm{~h}$ (Figure 3B). The relative expression of Bmserpin 2 in the midgut increased significantly at $24 \mathrm{~h}$, and its expression was significant until $72 \mathrm{hpi}$ (Figure 3C). Protein levels of Bmserpin2 in the haemolymph and midgut were upregulated after BmNPV infection (Figure 3D,E).
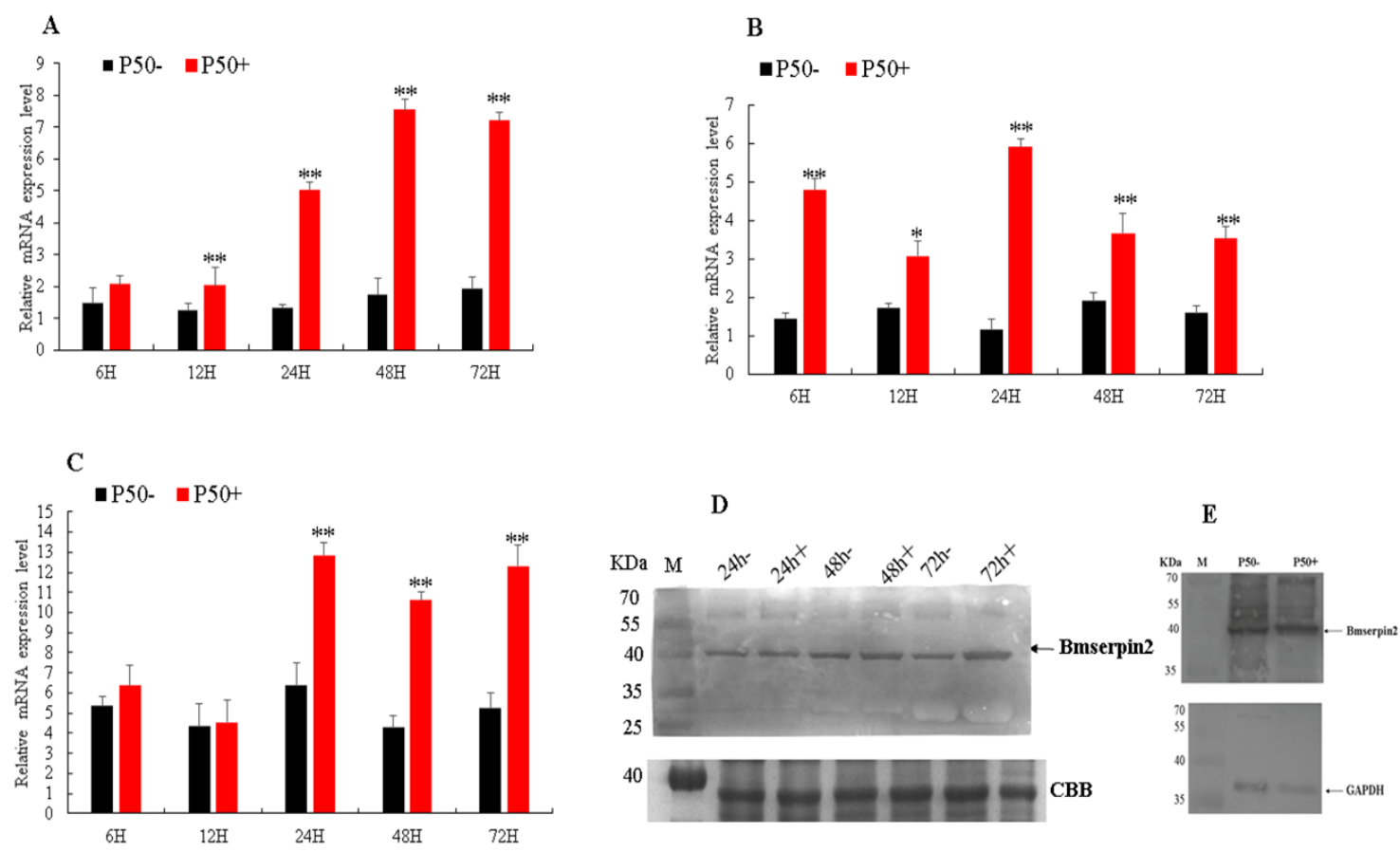

Figure 3. Expression profiles of the Bmserpin2 in P50 (susceptible strain) after Bombyx mori nucleopolyhedrovirus (BmNPV) infection in the haemocytes (A), fat body (B) and midgut (C) at $6,12,24,48$ and 72 hpi. P50- was used as a control. Relative expression levels were calculated using the $2^{-\Delta \Delta C t}$ method. Statistical analysis was performed using SPSS software (Version 19.0, Armonk, NY, USA). The significant differences are indicated by ${ }^{*}(p<0.05)$ or ${ }^{* *}(p<0.01)$. Western blot analyses were performed in haemolymph (D) at 24, 48 and $72 \mathrm{hpi}$, total proteins were coomassie brilliant blue (CBB)-stained as the loading control and in midgut (E) at $24 \mathrm{hpi}$. Anti-GAPDH was used as the loading control.

Bmserpin2 expression in BC9 after BmNPV infection revealed that its expression, compared to BC9- (control), was downregulated in BC9+ (infected) from 6 to $48 \mathrm{~h}$, however, its expression was significantly induced at $72 \mathrm{~h}$ in haemocytes (Figure $4 \mathrm{~A}$ ). The Bmserpin2 expression in the fat body was downregulated at the beginning (6-12 h) but increased gradually from $24 \mathrm{~h}$ and was significantly upregulated at $48 \mathrm{~h}$ (Figure 4B). In the midgut, Bmserpin 2 was downregulated until $24 \mathrm{~h}$, then significantly upregulated at 48 and $72 \mathrm{hpi}$ (Figure 4C). Furthermore, Western blot analyses indicated that protein levels of Bmserpin2 in the haemolymph and midgut of the BC9 strain were upregulated after BmNPV infection (Figure 4D,E). Therefore, the above results suggest that Bmserpin2 expression was induced in response to BmNPV infection and might play an important role in B. mori after BmNPV infection. 

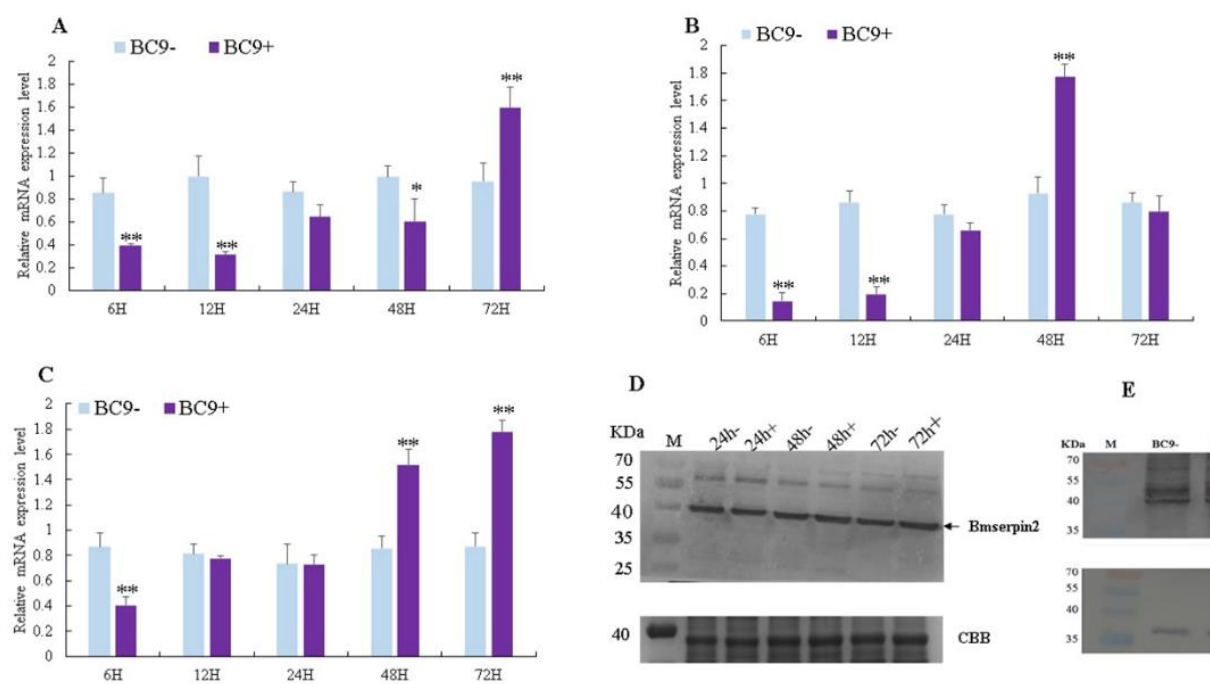

E

Figure 4. Expression profiles of the Bmserpin2 in BC9 (resistant strain) after BmNPV infection in the haemolymph (A), fat body (B) and midgut (C) at 6, 12, 24, 48 and 72 hpi. BC9- was used as a control. Relative expression levels were calculated using the $2^{-\Delta \Delta \mathrm{Ct}}$ method. Statistical analysis was performed using SPSS software. Significant differences are indicated by ${ }^{*}(p<0.05)$ or ${ }^{* *}(p<0.01)$. Western blot analyses were performed in haemolymph (D) 24,48 and $72 \mathrm{hpi}$, total proteins were Coomassie Brilliant Blue (CBB)-stained as the loading control and midgut (E) at $24 \mathrm{hpi}$. Anti-GAPDH was used as the loading control.

\subsection{Bmserpin2 Inhibits Melanization in Haemolymph}

The recombinant His-tagged Bmserpin2 was expressed with pET-28a in E. coli (BL21) competent cells. Expressed Bmserpin2 protein was successfully detected by SDS-PAGE with a molecular weight of $44.3 \mathrm{kDa}$ (Figure 5A). Western blotting was employed using anti-His antibodies which confirmed the target protein of $44.3 \mathrm{kDa}$ (Figure $5 \mathrm{~B}$ ).

A

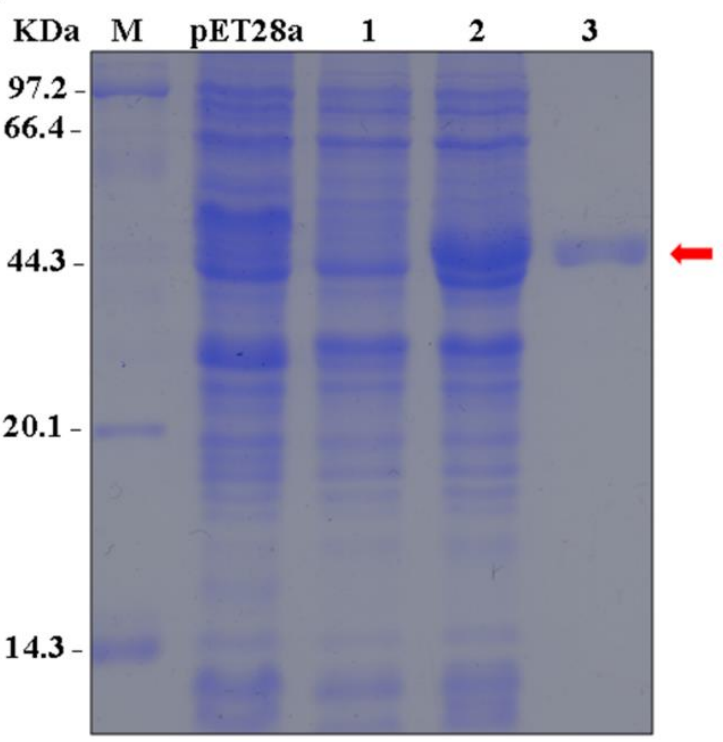

B

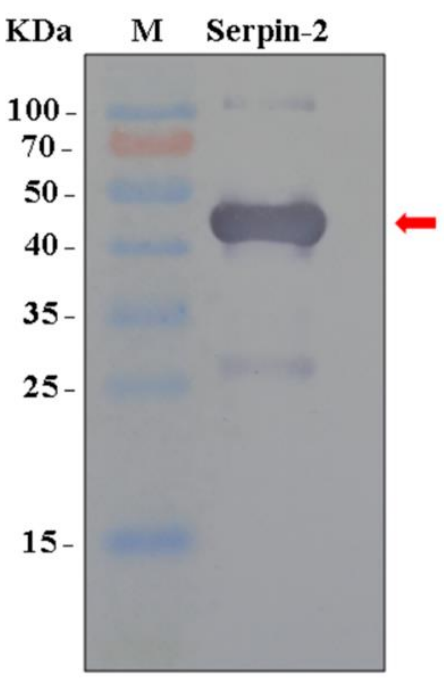

Figure 5. (A) Analysis of recombinant Bmserpin2 protein SDS-PAGE. M: molecular weight markers. pET-28a used as a control. Lane 1: Negative control without induction. Lane 2: Induced expression under isopropyl $\beta$-D-thiogalactoside (IPTG). Lane 3: Purified recombinant Bmserpin2 protein. (B) Western blot analysis of recombinant Bmserpin2 using anti-His antibody. 
To investigate the effect of Bmserpin2 on haemolymph melanization, cell-free haemolymph from P50 larvae was incubated with biologically active Bmserpin2. Haemolymph was exposed to air to observe spontaneous melanization. The colour of the haemolymph sample in the control group slowly became dark brown within 20 min. However, haemolymph mixed with Bmserpin2 (1-6 $\mu \mathrm{g})$ showed a decrease in melanization. This suggested that Bmserpin2 could inhibit the melanization in B. mori (Figure 6).

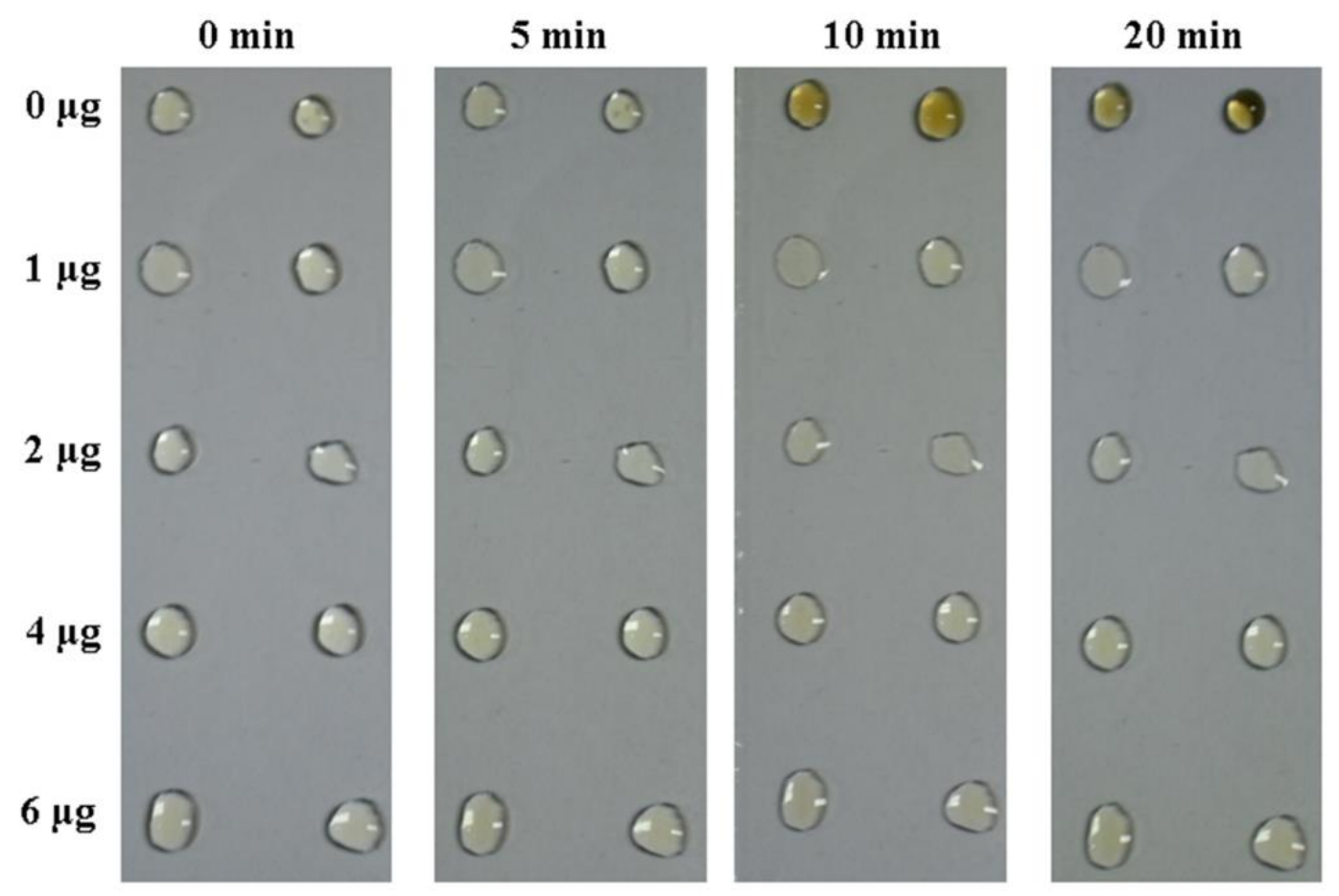

Figure 6. Bmserpin2 inhibits melanization in silkworm haemolymph. Cell-free haemolymph was mixed with different amounts of recombinant Bmserpin2. The control was prepared without Bmserpin2. The change in colour of haemolymph was observed and photographed.

\subsection{Silencing of Bmserpin2 in B. mori Increased PO Activity in Response to BmNPV Infection}

To further investigate melanization in silkworms after BmNPV infection, $\mathrm{PO}$ activity was assessed in cell-free haemolymph at $6,12,24,48$ and 72 hpi using the P50 strain. The results showed that PO activity in infected plasma did not show any changes at 6 and 12 hpi, however, as infection progressed, PO activity started to decrease at $24 \mathrm{hpi}$, and decreased remarkably at 48 and $72 \mathrm{hpi}$ as compared to control (Figure 7). To explore the effect of Bmserpin 2 on PO activity, we conducted knockdown of Bmserpin2 by injecting siRNA into 3rd day of fifth instar larvae. RNAi efficiency was detected by qPCR at 24 and $48 \mathrm{~h}$ after siRNA injection in haemocytes and fat body tissues, and indicated the transcript level of Bmserpin2 was greatly decreased (Figure 8). After $24 \mathrm{~h}$ injection of siRNA, cell-free haemolymph was collected from larvae and PO activity was measured. We found that knockdown of Bmserpin2 showed remarkable increase in PO activity than control after BmNPV infection (Figure 9). These results showed that Bmserpin2 could inhibit melanization by regulating PO activation. 


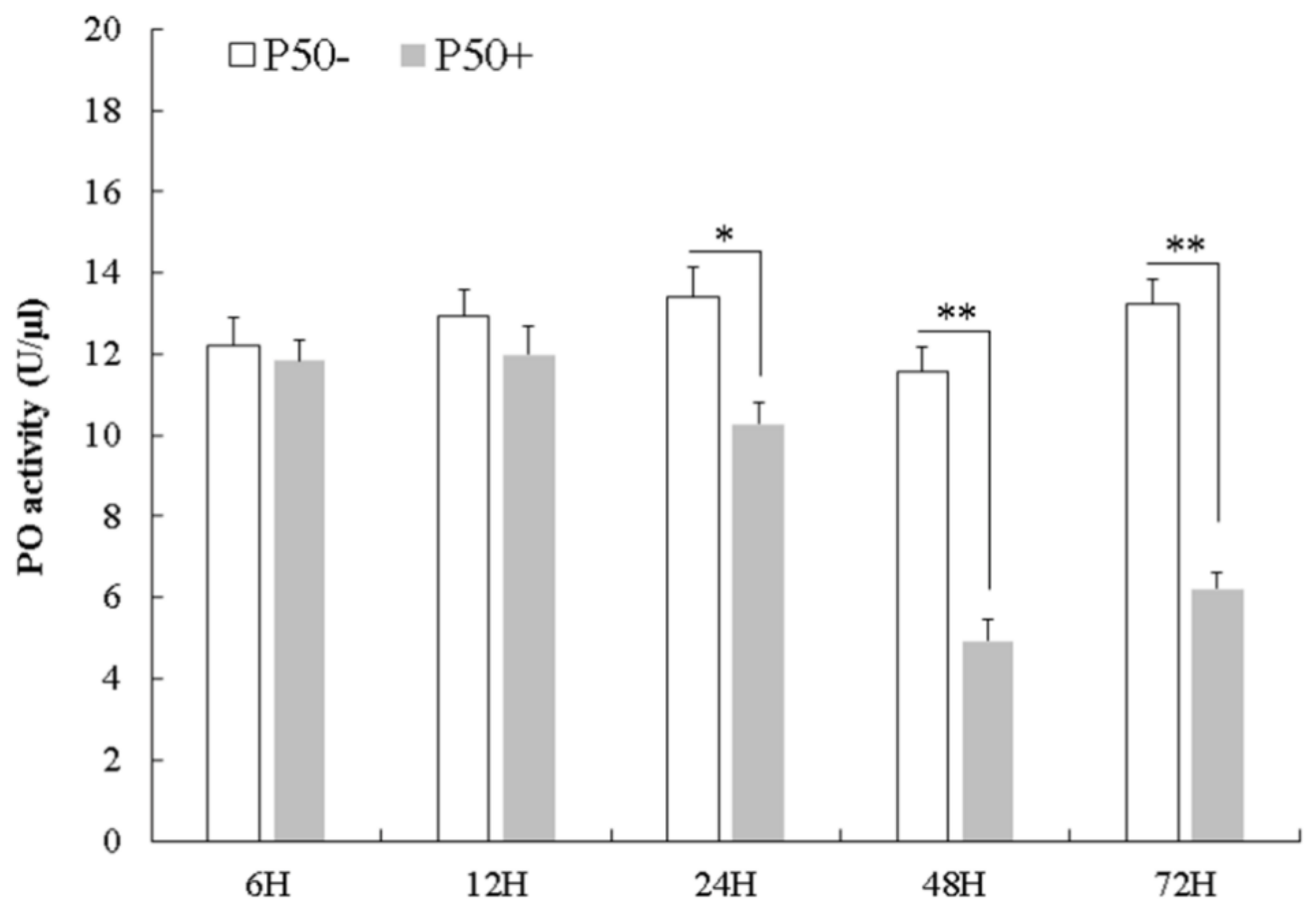

Figure 7. Phenoloxidase (PO) activity of cell-free haemolymph in control and infected B. mori at 6, 12, 24,48 and $72 \mathrm{hpi}$. Error bars show mean $\pm \mathrm{SD}(n=3)$. Statistical analysis was performed using SPSS software. Significant differences are indicated by ${ }^{*}(p<0.05)$ or ${ }^{* *}(p<0.01)$.

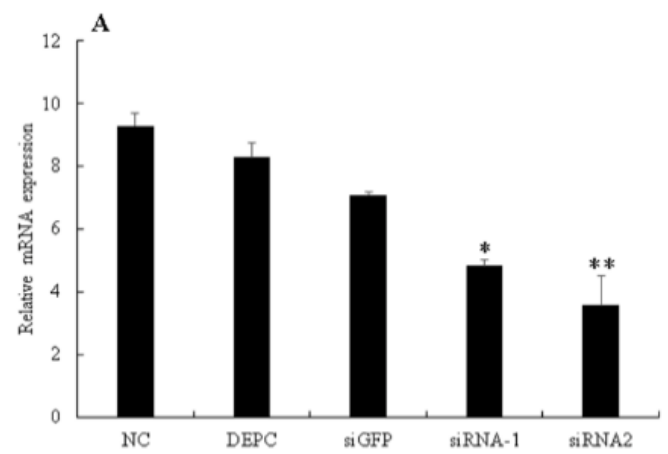

B
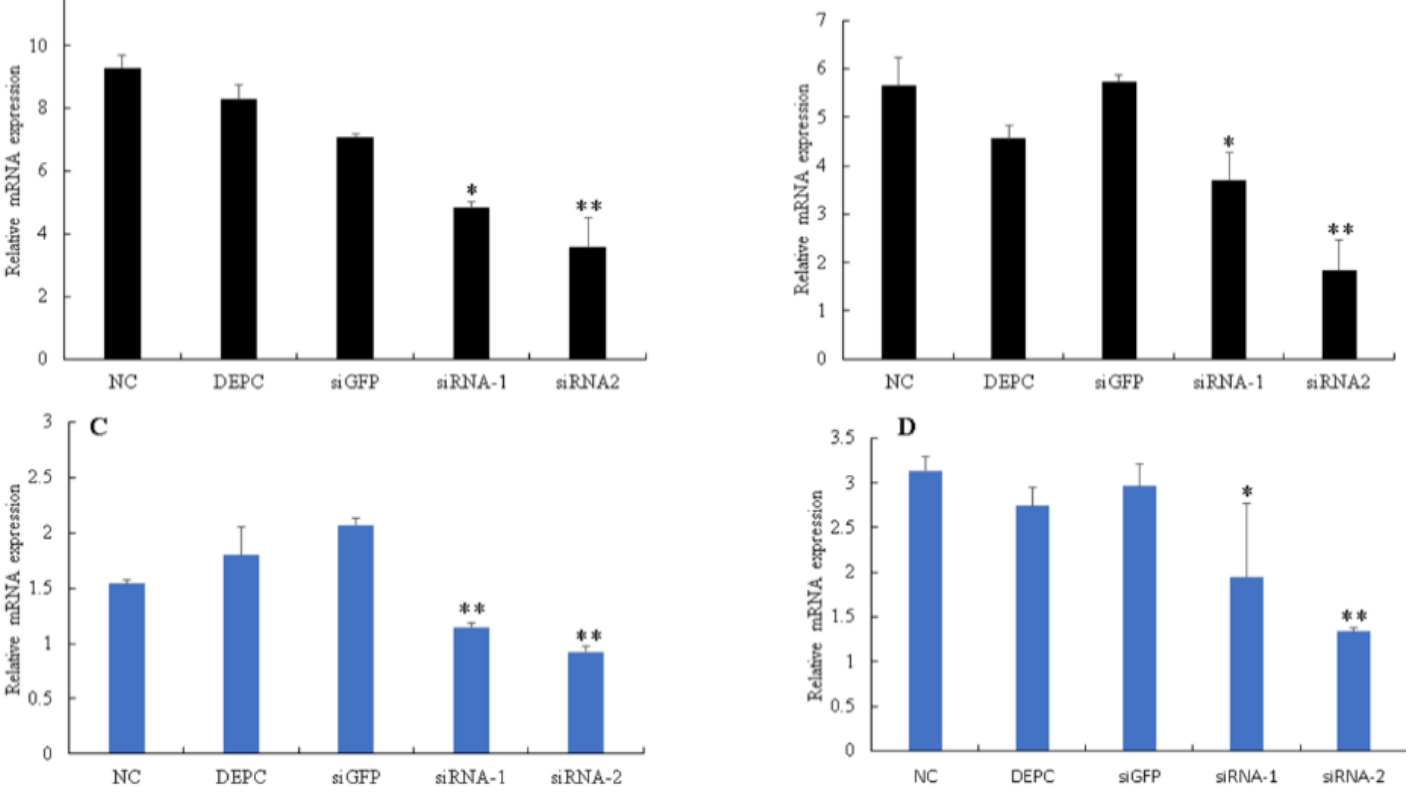

Figure 8. RNAi efficiency estimation of Bmserpin2. The expression level of Bmserpin2 in haemocytes and the fat body was measured at $24 \mathrm{~h}$ and $48 \mathrm{~h}$. Haemocytes (A,B); Fat body (C,D) after injection of siRNAs by qRT-PCR. NC: Negative control, DEPC: diethyl pyrocarbonate (DEPC) water, siGFP: si green fluorescent protein, siRNA-1: first target sequence of Bmserpin2 knockdown, siRNA-2: Second target sequence of Bmserpin2 knockdown. Statistical analysis was performed using SPSS software. Significant differences are indicated by * $(p<0.05)$ or ${ }^{* *}(p<0.01)$. 


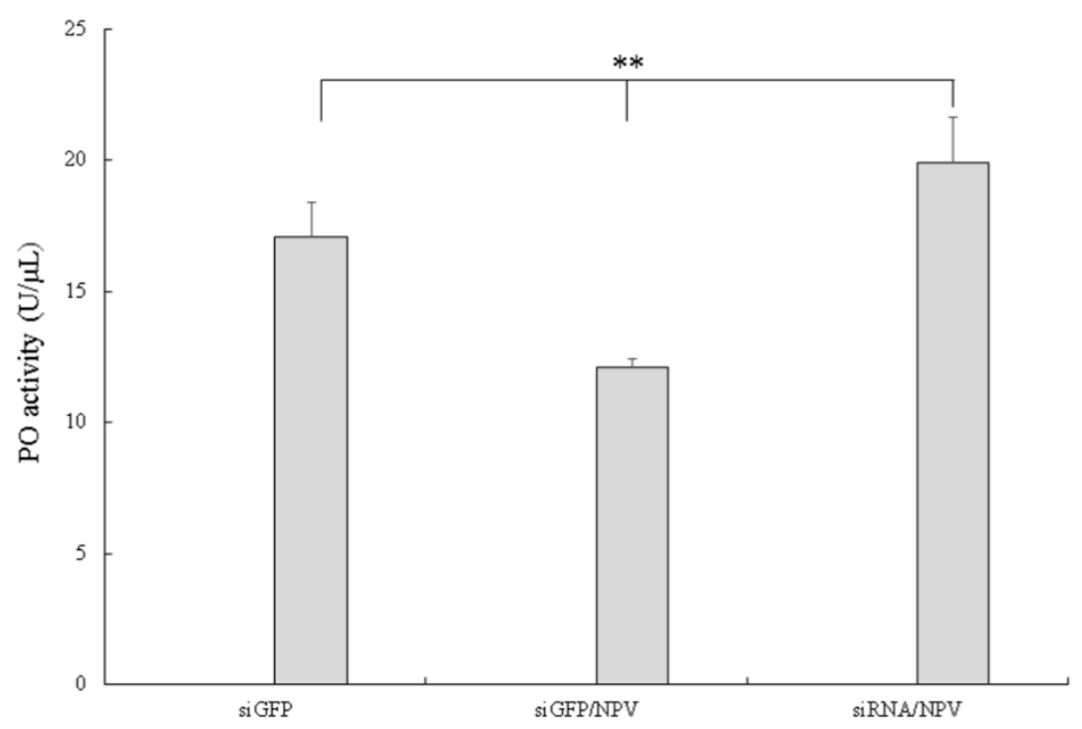

Figure 9. Knockdown of Bmserpin2 increased PO activity in cell-free haemolymph. PO activity was assayed using dopamine as a substrate, as described in "Materials and methods". Haemolymph collected from GFP (siGFP), GFP infected with BmNPV (siGFP/NPV) and Bmserpin2 siRNA (siRNA2 target sequence) infected with BmNPV (siRNA/NPV) was used to measure PO activity. Error bars show mean \pm SD $(n=3)$. Statistical analysis was performed using SPSS software. Significant differences are indicated by ${ }^{*}(p<0.05)$ or ${ }^{* *}(p<0.01)$.

\subsection{Virus Genomic DNA Copies Decreased in BmSerpin2-Depleted Haemolymph}

As knockdown of Bmserpin2 increased the PO activity after BmNPV infection, we next analysed virus genomic DNA copies in haemolymph after knockdown of Bmserpin2 at 24, 48 and $72 \mathrm{~h}$ post BmNPV infection. The results revealed that viral DNA copies were significantly reduced in contrast to siGFP (Figure 10). These results indicated that Bmserpin2 might be important for BmNPV to overcome host melanization.

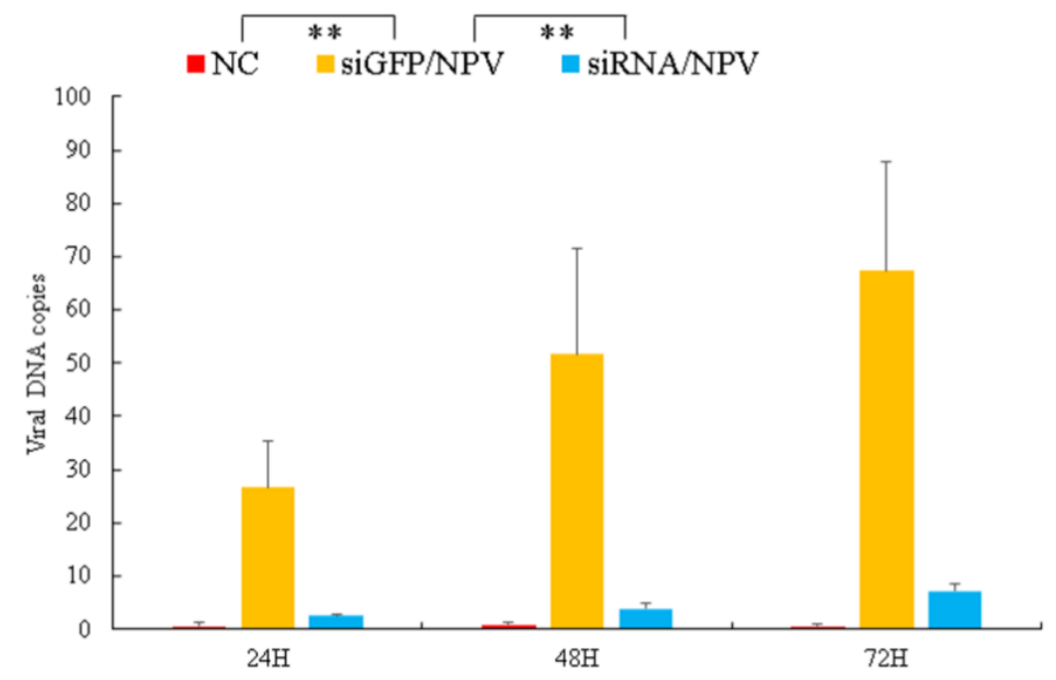

Figure 10. Viral genomic copies in Bmserpin2-depleted haemolymph. The BmNPV viral DNAs were extracted from haemolymph, and qPCR was performed to determine viral genomic DNA copies. Haemolymph from negative control (NC), GFP infected with BmNPV (siGFP/NPV) and Bmserpin2 siRNA infected with BmNPV (siRNA/NPV) were collected at 24, 48 and $72 \mathrm{~h}$ after BmNPV infection. Data were shown as mean \pm SD $(n=3)$. Statistical analysis was performed using SPSS software. Significant differences are indicated by ${ }^{*}(p<0.05)$ or ${ }^{* *}(p<0.01)$. 


\section{Discussion}

Serpins belong to the largest superfamily of serine protease inhibitors with wide range of physiological functions in insects. Serpins are involved in innate immunity by inhibiting the serine protease cascade that triggers melanization [14]. In previous research, many studies were conducted on serpins from B. mori in response to bacterial challenges $[24,26,28]$. We identified Bmserpin 2 from the transcriptome, which showed different expression in different resistant silkworm strains [33]. However, the role of B. mori serpins in response to BmNPV had not been conducted extensively.

Several studies have shown that serpins are expressed differentially in different tissues and developmental stages $[16,22,24]$. In the present study, we observed the expression of Bmserpin 2 in different tissues, and its expression was highest in the fat body. A similar expression of serpins has been characterised in different organisms, where it is also mainly expressed in the fat body which is responsible for production of most haemolymph proteins [23,26,40]. Meanwhile, we noted expression of Bmserpin2 in developmental stages, with it being highly expressed in 5th instar larvae. Likewise, Yang et al. noted an increased transcriptional level of serpin-12 in 5th instar larvae of M. sexta [23]. The fat body is an important tissue involved in innate immunity and a source of haemolymph protein synthesis [41], and the development of resistance in silkworms increases as the larvae grows. Thus, these results suggest that Bmerpin2 may participate in the innate immunity of the silkworm.

The mRNA expression level of Bmserpin 2 in P50 and BC9 significantly increased in the haemocytes, fat body and midgut after BmNPV infection, and protein levels of Bmserpin2 were also increased in the haemolymph and midgut post BmNPV infection. For P50 strain, Bmserpin 2 was upregulated at 24, 48 and $72 \mathrm{~h}$ after infection in the haemocytes and midgut; however, its expression started to upregulate at $6 \mathrm{hpi}$ in the fat body. A previous study also reported similar results in response to NPV that serpin-28 expression upregulated at 6 hpi [42]. We assume that it may possible that the silkworm immune response activated earlier than virus proliferation. In contrast, Bmserpin 2 expression was upregulated at 48 and $72 \mathrm{hpi}$ in BC9. This showed that Bmserpin 2 expression was delayed until $48 \mathrm{~h}$ or $72 \mathrm{~h}$ in BC9. This indicates that BC9 resists BmNPV infection more efficiently than P50.

Previous reports have studied the serpin genes in insects and showed increased expression in response to pathogens $[22,26,40,43]$. Yuan et al. reported that Helicoverpa armigera serpins were upregulated after viral infection [32]. Therefore, we assume that induced expression of Bmserpin2 might also be involved in the regulation of PPO in silkworms after BmNPV infection.

Melanization is an important immune response in insects. It consists of a cascade of SPs to activate PPO pathway. Serpins act as inhibitor of SPs to regulate the activity of PPO [14]. Previous studies have proven that serpins are involved in the regulation of the PPO cascade [21,24,44]. Several studies have reported that melanization is involved in antiviral immunity $[32,45,46]$. In M. sexta, epidermal growth factor-like motif (Egf) proteins (Egf1.0 and Egf1.5) are small serine proteinase inhibitors that inhibit melanization by regulating PPO [47,48]. Recently, research has shown that melanization is inhibited in silkworms after BmNPV infection [49]. In our study, we found that the Bmserpin2 response to NPV infection, and PO activity in haemolymph was drastically decreased after BmNPV infection. Yuan et al., also showed that after virus infection PO activity was decreased [32]. However, another study found that PO activity increased in a susceptible strain (871) after infection of BmNPV (BV) [49]. As the ODV and BV infection mechanisms are different, so we assume that this might account for changes in PO activity. After knockdown of Bmserpin2, the PO activity was higher than the control (iGFP/NPV). In particular, the viral genomic DNA copies were significantly reduced in Bmserpin2 depleted haemolymph. Yuan et al. had shown that knockdown of H. armigera serpin-9 and serpin-5 increased PO activity, and the virus load was decreased [32]. In our study, Bmserpin 2 showed different expression in P50 and BC9 strains, and knockdown of Bmserpin 2 caused an increase in PO activity. We speculate that BmNPV inhibits host melanization by regulating Bmserpin2 expression. In the light of these results, we propose a model for the role of Bmserpin 2 in the PO cascade of B. mori after BmNPV infection (Figure 11). Once BmNPV penetrates the midgut and enters the fat body, then the fat body will synthesise immune related proteins including Bmserpin2, which will be secreted 
into the haemolymph. In the haemolymph, the PO cascade will be initiated via serine proteases to produce melanin to kill BmNPV. In successful BmNPV proliferation, the virus will overcome the host PO response through upregulating Bmserpin2, which eventually will inhibit serine protease to regulate the PO cascade. The aforementioned results manifested that Bmserpin 2 could suppress melanization by regulating PPO after BmNPV infection.

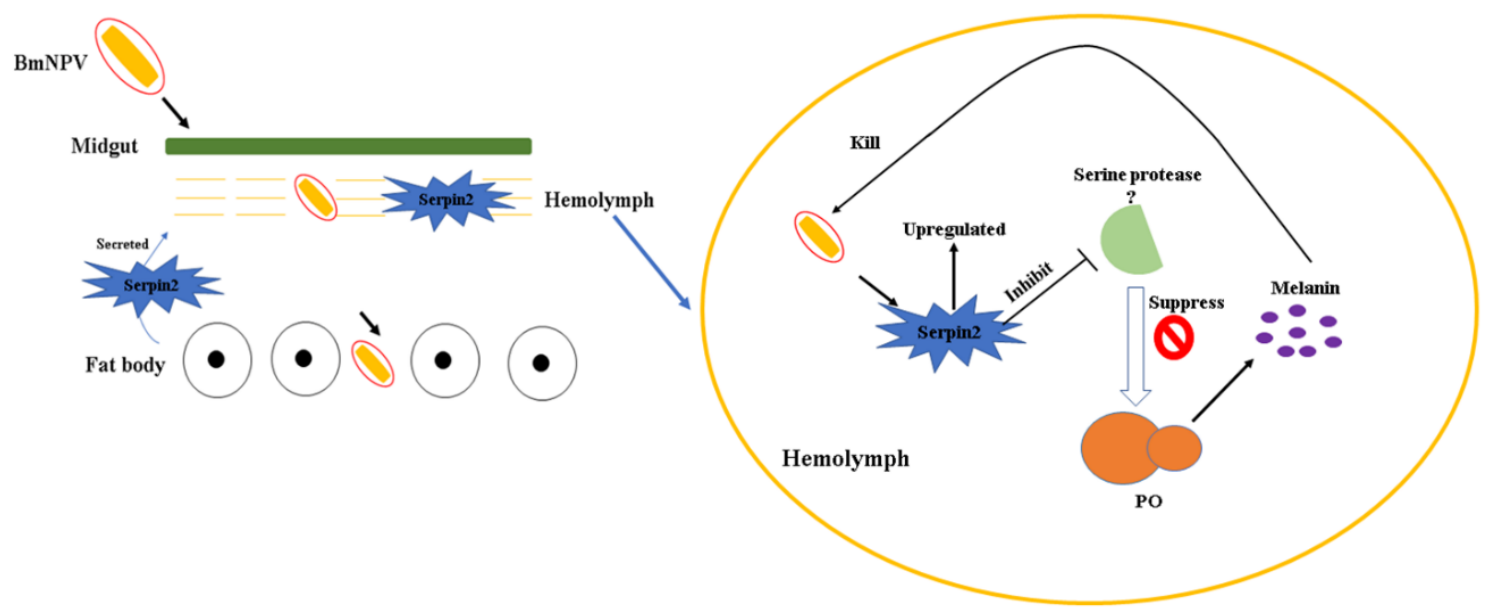

Figure 11. A proposed diagram of role of Bmserpin2 in the regulation of the PO cascade after BmNPV infection.

\section{Conclusions}

In conclusion, we have characterised Bmserpin2 in response to BmNPV infection. Bmserpin2 expression was significantly up-regulated in different resistant strains after BmNPV infection. PO activity was down-regulated following BmNPV infection and Bmserpin2 showed an inhibition of melanization in the haemolymph. Knockdown of Bmserpin2 resulted in a dramatic increase in PO activity. However, some questions remain to be answered; for instance, it is not clear which of these SPs is the target of Bmserpin2 inhibition. Further work is underway in our laboratory to investigate the interaction between the host PPO pathway and BmNPV. These findings provide an insight into the innate immunity in silkworms and its mechanism in response to BmNPV infection.

Author Contributions: Conceptualization, S.T. and J.-P.X.; Data curation, S.T.; Investigation, S.T.; methodology, S.T. and J.W.; resources, S.-Z.Z., B.L., P.H., L.-B.Z. and L.-L.Y.; supervision, J.-P.X.; writing-review and editing, S.T. and J.-P.X.

Funding: This work was supported by the Natural Science Foundation of China (No. 31973002), International cooperation project of Anhui province (No. 1804b06020345), and Shahzad Toufeeq thanks the China Scholarship Council (CSC).

Conflicts of Interest: The authors declare no conflict of interest.

\section{References}

1. Mita, K.; Kasahara, M.; Sasaki, S.; Nagayasu, Y.; Yamada, T.; Kanamori, H.; Namiki, N.; Kitagawa, M.; Yamashita, H.; Yasukochi, Y.; et al. The genome sequence of silkworm, Bombyx mori. DNA Res. 2004, 11, 27-35. [CrossRef] [PubMed]

2. Blissard, G.W.; Rohrmann, G.F. Baculovirus diversity and molecular biology. Ann. Rev. Entomol. 1990, 35, 127-155. [CrossRef] [PubMed]

3. Yao, Q.; Li, M.W.; Wang, Y.; Wang, W.B.; Lu, J.; Dong, Y.; Chen, K.P. Screening of molecular markers for NPV resistance in Bombyx mori L. (Lep., Bombycidae). J. Appl. Entomol. 2003, 127, 134-136. [CrossRef]

4. Bao, Y.-Y.; Tang, X.-D.; Lv, Z.-Y.; Wang, X.-Y.; Tian, C.-H.; Xu, Y.P.; Zhang, C.-X. Gene expression profiling of resistant and susceptible Bombyx mori strains reveals nucleopolyhedrovirus-associated variations in host gene transcript levels. Genomics 2009, 94, 138-145. [CrossRef] [PubMed] 
5. Lavine, M.D.; Strand, M.R. Insect hemocytes and their role in immunity. Insect Biochem. Mol. Biol. 2002, 32, 1295-1309. [CrossRef]

6. Lemaitre, B.; Hoffmann, J. The Host defense of drosophila melanogaster. Annu. Rev. Immunol. 2007, 25, 697-743. [CrossRef] [PubMed]

7. Steinert, S.; Levashina, E.A. Intracellular immune responses of dipteran insects. Immunol. Rev. 2011, 240, 129-140. [CrossRef] [PubMed]

8. Jiang, H.; Wang, Y.; Yu, X.-Q.; Zhu, Y.; Kanost, M. Prophenoloxidase-activating proteinase-3 (PAP-3) from Manduca sexta hemolymph: A clip-domain serine proteinase regulated by serpin-1J and serine proteinase homologs. Insect Biochem. Mol. Biol. 2003, 33, 1049-1060. [CrossRef]

9. Kanost, M.R.; Gorman, M.J. 4-phenoloxidases in insect immunity. In Insect Immunology; Beckage, N.E., Ed.; Academic Press: San Diego, CA, USA, 2008; pp. 69-96. [CrossRef]

10. Lu, A.; Zhang, Q.; Zhang, J.; Yang, B.; Wu, K.; Xie, W.; Luan, Y.X.; Ling, E. Insect prophenoloxidase: The view beyond immunity. Front. Physiol. 2014, 5, 252. [CrossRef] [PubMed]

11. Kanost, M.R. Serine proteinase inhibitors in arthropod immunity. Dev. Comp. Immunol. 1999, 23, $291-301$. [CrossRef]

12. Gettins, P.G. Serpin structure, mechanism, and function. Chem. Rev. 2002, 102, 4751-4804. [CrossRef] [PubMed]

13. Huntington, J.A. Serpin structure, function and dysfunction. J. Thromb. Haemost. 2011, 9, 26-34. [CrossRef] [PubMed]

14. Meekins, D.A.; Kanost, M.R.; Michel, K. Serpins in arthropod biology. Semin. Cell Dev. Biol. 2017, 62, $105-119$. [CrossRef] [PubMed]

15. Garrett, M.; Fullaondo, A.; Troxler, L.; Micklem, G.; Gubb, D. Identification and analysis of serpin-family genes by homology and synteny across the 12 sequenced Drosophilid genomes. BMC Genom. 2009, 10, 489. [CrossRef] [PubMed]

16. Zou, Z.; Picheng, Z.; Weng, H.; Mita, K.; Jiang, H. A comparative analysis of serpin genes in the silkworm genome. Genomics 2009, 93, 367-375. [CrossRef] [PubMed]

17. Li, M.; Christen, J.M.; Dittmer, N.T.; Cao, X.; Zhang, X.; Jiang, H.; Kanost, M.R. The Manduca sexta serpinome: Analysis of serpin genes and proteins in the tobacco hornworm. Insect Biochem. Mol. Biol. 2018, 102, 21-30. [CrossRef] [PubMed]

18. Xiong, G.H.; Xing, L.S.; Lin, Z.; Saha, T.T.; Wang, C.; Jiang, H.; Zou, Z. High throughput profiling of the cotton bollworm Helicoverpa armigera immunotranscriptome during the fungal and bacterial infections. BMC Genomics 2015, 16, 321. [CrossRef] [PubMed]

19. Jiang, R.; Zhang, B.; Kurokawa, K.; So, Y.-I.; Kim, E.-H.; Hwang, H.O.; Lee, J.-H.; Shiratsuchi, A.; Zhang, J.; Nakanishi, Y.; et al. 93-kDa twin-domain serine protease inhibitor (serpin) has a regulatory function on the beetle toll proteolytic signaling cascade. J. Biol. Chem. 2011, 286, 35087-35095. [CrossRef] [PubMed]

20. Zou, Z.; Evans, J.D.; Lu, Z.; Zhao, P.; Williams, M.; Sumathipala, N.; Hetru, C.; Hultmark, D.; Jiang, H. Comparative genomic analysis of the Tribolium immune system. Genome Biol. 2007, 8, R177. [CrossRef] [PubMed]

21. Zhu, Y.; Wang, Y.; Gorman, M.J.; Jiang, H.; Kanost, M.R. Manduca sexta serpin-3 regulates prophenoloxidase activation in response to infection by inhibiting prophenoloxidase-activating proteinases. J. Biol. Chem. 2003, 278, 46556-46564. [CrossRef] [PubMed]

22. Suwanchaichinda, C.; Ochieng, R.; Zhuang, S.; Kanost, M.R. Manduca sexta serpin-7, a putative regulator of hemolymph prophenoloxidase activation. Insect Biochem. Mol. Biol. 2013, 43, 555-561. [CrossRef] [PubMed]

23. Yang, F.; Wang, Y.; Sumathipala, N.; Cao, X.; Kanost, M.R.; Jiang, H. Manduca sexta serpin-12 controls the prophenoloxidase activation system in larval hemolymph. Insect Biochem. Mol. Biol. 2018, 99, 27-36. [CrossRef] [PubMed]

24. Li, J.; Ma, L.; Lin, Z.; Zou, Z.; Lu, Z. Serpin-5 regulates prophenoloxidase activation and antimicrobial peptide pathways in the silkworm, Bombyx mori. Insect Biochem. Mol. Biol. 2016, 73, 27-37. [CrossRef] [PubMed]

25. Li, B.; Yu, H.-Z.; Ye, C.-J.; Ma, Y.; Li, X.; Fan, T.; Chen, F.-S.; Xu, J.-P. Bombyx mori Serpin6 regulates prophenoloxidase activity and the expression of antimicrobial proteins. Gene 2017, 610, 64-70. [CrossRef] [PubMed] 
26. Liu, D.; Wang, L.; Yang, L.; Qian, C.; Wei, G.; Dai, L.; Li, J.; Zhu, B.; Liu, C. Serpin-15 from Bombyx mori inhibits prophenoloxidase activation and expression of antimicrobial peptides. Dev. Comp. Immunol. 2015, 51, 22-28. [CrossRef] [PubMed]

27. Guo, P.-C.; Dong, Z.; Zhao, P.; Zhang, Y.; He, H.; Tan, X.; Zhang, W.; Xia, Q. Structural insights into the unique inhibitory mechanism of the silkworm protease inhibitor serpin18. Sci. Rep. 2015, 5, 11863. [CrossRef] [PubMed]

28. Wang, L.; Liu, H.; Fu, H.; Zhang, L.; Guo, P.; Xia, Q.; Zhao, P. Silkworm serpin32 functions as a negative-regulator in prophenoloxidase activation. Dev. Comp. Immunol. 2019, 91, 123-131. [CrossRef] [PubMed]

29. Shelby, K.S.; Popham, H.J. Plasma phenoloxidase of the larval tobacco budworm, Heliothis virescens, is virucidal. J. Insect Sci. (Online) 2006, 6, 1-12. [CrossRef] [PubMed]

30. Zhao, P.; Lu, Z.; Strand, M.R.; Jiang, H. Antiviral, anti-parasitic, and cytotoxic effects of 5,6-dihydroxyindole (DHI), a reactive compound generated by phenoloxidase during insect immune response. Insect Biochem. Mol. Biol. 2011, 41, 645-652. [CrossRef] [PubMed]

31. Rodriguez-Andres, J.; Rani, S.; Varjak, M.; Chase-Topping, M.E.; Beck, M.H.; Ferguson, M.C.; Schnettler, E.; Fragkoudis, R.; Barry, G.; Merits, A.; et al. Phenoloxidase activity acts as a mosquito innate immune response against infection with Semliki Forest virus. PLoS Pathog. 2012, 8, e1002977. [CrossRef] [PubMed]

32. Yuan, C.; Xing, L.; Wang, M.; Wang, X.; Yin, M.; Wang, Q.; Hu, Z.; Zou, Z. Inhibition of melanization by serpin-5 and serpin-9 promotes baculovirus infection in cotton bollworm Helicoverpa armigera. PLoS Pathog. 2017, 13, e1006645. [CrossRef] [PubMed]

33. Wang, X.-Y.; Yu, H.-Z.; Geng, L.; Xu, J.-P.; Yu, D.; Zhang, S.-Z.; Ma, Y.; Fei, D.-Q. Comparative transcriptome analysis of Bombyx mori (Lepidoptera) larval midgut response to bmnpv in susceptible and near-isogenic resistant strains. PLoS ONE 2016, 11, e0155341. [CrossRef] [PubMed]

34. Rahman, M.M.; Gopinathan, K.P. Systemic and in vitro infection process of Bombyx mori nucleopolyhedrovirus. Virus Res. 2004, 101, 109-118. [CrossRef] [PubMed]

35. Yu, H.; Wang, X.; Xu, J.; Ma, Y.; Zhang, S.; Yu, D.; Fei, D.; Muhammad, A. iTRAQ-based quantitative proteomics analysis of molecular mechanisms associated with Bombyx mori (Lepidoptera) larval midgut response to BmNPV in susceptible and near-isogenic strains. J. Proteom. 2017, 165, 35-50. [CrossRef] [PubMed]

36. Shahzad, T.; Zhan, M.-Y.; Yang, P.-J.; Yu, X.-Q.; Rao, X.-J. Molecular cloning and analysis of a C-type lectin from silkworm Bombyx mori. Arch. Insect Biochem. Physiol. 2017, 95, e21391. [CrossRef] [PubMed]

37. Livak, K.J.; Schmittgen, T.D. Analysis of relative gene expression data using real-time quantitative PCR and the $2^{-\Delta \Delta C T}$ method. Methods 2001, 25, 402-408. [CrossRef] [PubMed]

38. Huang, S.; Yao, L.; Zhang, J.; Huang, L. Direct and indirect effects of RNA interference against pyridoxal kinase and pyridoxine 5'-phosphate oxidase genes in Bombyx mori. Gene 2016, 587, 48-52. [CrossRef] [PubMed]

39. Bao, Y.Y.; Lv, Z.Y.; Liu, Z.B.; Xue, J.; Xu, Y.P.; Zhang, C.X. Comparative analysis of Bombyx mori nucleopolyhedrovirus responsive genes in fat body and haemocyte of $B$. mori resistant and susceptible strains. Insect Mol. Biol. 2010, 19, 347-358. [CrossRef] [PubMed]

40. Kausar, S.; Abbas, M.N.; Qian, C.; Zhu, B.; Sun, Y.; Sun, Y.; Wang, L.; Wei, G.; Maqsood, I.; Liu, C.-L. Serpin-14 negatively regulates prophenoloxidase activation and expression of antimicrobial peptides in Chinese oak silkworm Antheraea pernyi. Dev. Comp. Immunol. 2017, 76, 45-55. [CrossRef] [PubMed]

41. Ferrandon, D.; Imler, J.-L.; Hetru, C.; Hoffmann, J.A. The Drosophila systemic immune response: Sensing and signalling during bacterial and fungal infections. Nat. Rev. Immunol. 2007, 7, 862. [CrossRef] [PubMed]

42. Gao, Q.; Yang, L.; Dai, J.; Yuan, G.; Wang, L.; Qian, C.; Zhu, B.; Liu, C.; Wei, G. Characterization and functional analysis of serpin-28 gene from silkworm, Bombyx mori. J. Invertebr. Pathol. 2018, 159, 18-27. [CrossRef] [PubMed]

43. An, C.; Kanost, M.R. Manduca sexta serpin-5 regulates prophenoloxidase activation and the Toll signaling pathway by inhibiting hemolymph proteinase HP6. Insect Biochem. Mol. Biol. 2010, 40, 683-689. [CrossRef] [PubMed]

44. Chu, Y.; Zhou, F.; Liu, Y.; Hong, F.; Wang, G.; An, C. Ostrinia furnacalis serpin-3 regulates melanization cascade by inhibiting a prophenoloxidase-activating protease. Insect Biochem. Mol. Biol. 2015, 61, 53-61. [CrossRef] [PubMed] 
45. Clark, K.D. Altered tyrosine metabolism and melanization complex formation underlie the developmental regulation of melanization in Manduca sexta. Insect Biochem. Mol. Biol. 2015, 58, 66-75. [CrossRef] [PubMed]

46. Sangsuriya, P.; Charoensapsri, W.; Sutthangkul, J.; Senapin, S.; Hirono, I.; Tassanakajon, A.; Amparyup, P. A novel white spot syndrome virus protein WSSV164 controls prophenoloxidases, PmproPOs in shrimp melanization cascade. Dev. Comp. Immunol. 2018, 86, 109-117. [CrossRef] [PubMed]

47. Lu, Z.; Beck, M.H.; Wang, Y.; Jiang, H.; Strand, M.R. The viral protein Egf1.0 is a dual activity inhibitor of prophenoloxidase-activating proteinases 1 and 3 from Manduca sexta. J. Biol. Chem. 2008, 283, 21325-21333. [CrossRef] [PubMed]

48. Lu, Z.; Beck, M.H.; Strand, M.R. Egf1.5 is a second phenoloxidase cascade inhibitor encoded by Microplitis demolitor bracovirus. Insect Biochem. Mol. Biol. 2010, 40, 497-505. [CrossRef] [PubMed]

49. Chen, T.-T.; Hu, N.; Tan, L.-R.; Xiao, Q.; Dong, Z.-Q.; Chen, P.; Xu, A.-Y.; Pan, M.-H.; Lu, C. Resistant silkworm strain block viral infection independent of melanization. Pestic. Biochem. Physiol. 2019, 154, 88-96. [CrossRef] [PubMed]

(C) 2019 by the authors. Licensee MDPI, Basel, Switzerland. This article is an open access article distributed under the terms and conditions of the Creative Commons Attribution (CC BY) license (http://creativecommons.org/licenses/by/4.0/). 